Hitchman, E., Hitchman, R. and King, L. (2017) 'BacMam delivery of a protective gene to reduce renal ischemiareperfusion injury', Human Gene Therapy

DOI: https://doi.org/10.1089/hum.2016.100

This document is the authors' Accepted Manuscript.

License: https://creativecommons.org/licenses/by-nc-nd/4.0

Available from RADAR: https://radar.brookes.ac.uk/radar/items/e99d7036-617a-475f-a383-8fa844112db5/1/

Copyright (C) and Moral Rights are retained by the author(s) and/ or other copyright owners unless otherwise waved in a license stated or linked to above. A copy can be downloaded for personal non-commercial research or study, without prior permission or charge. This item cannot be reproduced or quoted extensively from without first obtaining permission in writing from the copyright holder(s). The content must not be changed in any way or sold commercially in any format or medium without the formal permission of the copyright holders. 


\section{BacMam delivery of a protective gene to reduce renal ischemia-reperfusion injury}

Elisabetta Hitchman ${ }^{1,3}$, Richard B Hitchman ${ }^{2,4}$, Linda A King ${ }^{1}$

${ }^{1}$ Department of Biological and Medical Sciences, Faculty of Health \& Life Sciences, Oxford Brookes University, Oxford OX3 OBP, UK.

${ }^{2}$ Oxford Expression Technologies Ltd, Biolnnovation Hub, Gipsy Lane, Oxford OX3 OBP UK ${ }^{3}$ Current address: Oxford BioMedica (UK) Ltd, Medawar Centre, Robert Robinson Avenue, The Oxford Science Park, Oxford, OX4 4GA, UK.

${ }^{4}$ Current address: Structural Biology, Evotec Ltd (UK), 114 Innovation Drive, Milton Park, Abingdon, OX14 4SA, UK.

Correspondence: Prof. Linda A King, Department of Biological and Medical Sciences, Oxford Brookes University, Oxford OX3 OBP, UK.

E-mail: laking@brookes.ac.uk

Phone: $+44(1865) 483241$ 


\section{Abstract}

Ischaemia-reperfusion $(I / R)$ injury remains the primary contributor to delayed graft function in kidney transplantation. The beneficial application of manganese superoxide dismutase (sod), delivered by a BacMam vector, against renal I/R injury has not been evaluated previously. Therefore, in this study we overexpressed sod-2 in proximal tubular epithelial (HK-2) cells and porcine kidney organs during simulated renal I/R injury.

Incubation of HK-2 cells with antimycin A and 2-deoxyglucose resulted in a significant decrease in intracellular ATP levels; following reperfusion, ATP levels significantly increased over time in cells overexpressing sod-2. In addition, lactate dehydrogenase (LDH) release declined over $72 \mathrm{~h}$ in BacMam-transduced injured cells. Ex vivo delivery of sod-2 significantly increased ATP levels in organs after $24 \mathrm{~h}$ of cold perfusion.

In vitro and ex vivo results suggested that BacMam transduction successfully delivered sod2, which reduced injury associated with $\mathrm{I} / \mathrm{R}$, by improving ATP cell content and decreasing $\mathrm{LDH}$ release with a subsequent increase in kidney tissue viability. These data provide further evidence for the potential application of BacMam as a gene delivery system for attenuating injury after cold preservation. 


\section{Introduction}

Ischemia followed by reperfusion (I/R) is a pivotal mechanism of organ injury in various medical conditions ${ }^{1}$. Clinically, $\mathrm{I} / \mathrm{R}$ injury is associated with delayed graft function, graft rejection, chronic rejection and chronic graft dysfunction ${ }^{1}$. I/R injury is characterized by restriction of blood supply to an organ followed by restoration of blood flow and reoxygenation. This can results in a severe hypoxic state (prolonged insufficient flow of nutrients and oxygen to the organ) activating cell death programs ${ }^{1}$. Renal hypoxia injury has been shown to be related to a number of cellular biochemical, physiological and morphological alterations, including increased reactive oxygen species (ROS). In particular hypoxia leads to a severe depletion of intracellular ATP that causes the activation of apoptotic pathways $s^{4,5}$.

I/R injury still remains a major cause of acute kidney injury (AKI) resulting in high levels of morbidity and mortality ${ }^{2}$ and is a major determinant of graft survival in kidney transplantation $^{3}$. Two types of ischaemia may occur during renal transplantation, warm and cold ischaemia. Warm ischaemia is further divided into donor warm ischaemia (either after circulatory death or after brain death) and recipient warm ischaemia. Cold ischaemia occurs when kidneys collected from living donors are directly placed in cold preservation solution. The work described in this study sought to examine the possibility of reducing injury associated with cold preservation.

Emerging strategies tried to prevent renal I/R injury by preserving ATP levels in an attempt to improve short- and long-term outcomes following kidney transplantation ${ }^{6,7,8,9}$. In vitro models are generally used to study molecular mechanisms, specific pathways involved in cellular damage or death caused by I/R injury events; they provide evidence for further in vivo analysis. However, as cell-based experimental models mimic only some of the features of tissue ischaemia and I/R injury, they cannot reproduce the in vivo tissue environment completely ${ }^{10}$. The major areas of in vitro $\mathrm{I} / \mathrm{R}$ injury investigation have included 
overexpression of protective genes, enhancing enzyme activity and suppressing the expression of genes that activate the cell death pathway. Because low blood supply during I/R injury leads to the deprival of ATP concentration, most cell-based models of I/R have focussed on inhibiting the production or synthesis of ATP through chemical ${ }^{11}$, enzymatic ${ }^{12}$ or anaerobic chamber ${ }^{13}$ approaches $^{14}$. In the present study, we used a chemical approach to induce $I / R$ injury in an immortalized proximal tubule epithelial cell line (HK-2) from normal adult human kidney.

Mitochondria are the main cellular sites of radical oxygen species production; consequently, they represent an early target of oxygen injury via enhanced production of ROS, followed by mitochondrial dysfunction ${ }^{15}$. Manganese superoxide dismutase (SOD-2) is the major antioxidant in the mitochondria; it localizes in the mitochondrial matrix ${ }^{16}$ and exists as a homotetramer containing one $\mathrm{Mn}$ (Manganese) atom per subunit ${ }^{8}$. Its main function is to catalyse the dismutation of the superoxide anion $\left(\mathrm{O}_{2}{ }^{--}\right)$producing hydrogen peroxide $\left(\mathrm{H}_{2} \mathrm{O}_{2}\right)^{8}$. Studies, using a rodent model of renal I/R injury, showed endogenous SOD-2 was rapidly inactivated at early times during renal transplantation; suggesting that loss of SOD-2 activity may have played an important role in inducing further injury ${ }^{17,18,19}$, e.g. SOD-2 deficiency in mice leads to prenatal lethality ${ }^{15}$. Furthermore, a number of reports have shown that high levels of SOD-2 play a key role in attenuating injury or death under conditions of enhanced oxidative stress ${ }^{21,22,23}$.

To date, more than $70 \%$ of clinical trials and gene therapy studies have been carried out using mammalian virus vectors such as adenovirus ${ }^{24,25}$. BacMam vectors (baculovirus-based vectors for expression in mammalian cells) provide an attractive alternative allowing scalable virus production ${ }^{26}$.

A number of studies have reported that the mode and kinetics of BacMam entry into mammalian cells is identical to that found in insect cells $s^{27,28}$. Baculovirus enters insect cells by receptor-mediated endocytosis, followed by $\mathrm{pH}$-dependent fusion of the envelope with the 
endosome $^{27,28}$. BacMam vectors also enter mammalian cells via endocytosis and are released into the cytoplasm by acid-induced fusion of the envelope with the endosomal membrane. Studies have suggested that the rod shaped nucleocapsid, containing the virus genome, is then transported into the nucleus through a nuclear pore. ${ }^{29}$. The prototype baculovirus used for BacMam studies is Autographa californica multiple nucleopolyhedrovirus (AcMNPV) ${ }^{22}$. High-levels of expression have been observed in transduced vertebrate cells and these vectors have low cytotoxicity, high insert size capacity and a relatively low immune-response profile compared to mammalian virus-vectors $23,26,30,31,32,33$. A number of studies have reported that baculovirus stimulates an immuneresponse in mammalian cells. It was demonstrated that baculovirus possess an adjuvant activity that promotes humoral and cellular immune responses against co-administered antigens, maturation of dendritic cells (DCs), and production of pro-inflammatory cytokines $^{34,35,36,37,38}$. Indeed, our previous studies have suggested that BacMam can regulate the cellular immune-response by up-regulating a number of cytokines and other immuneresponse genes after in vitro transduction into HEK293 cells. However, it should be noted that no apparent cellular injury or reduction in cell viability was observed after in vitro and ex vivo solid organ transduction ${ }^{39}$. Therefore, adaptive and innate immunity should be taken into consideration when designing the BacMam gene delivery approach.

The purpose of this study was to investigate BacMam as a novel gene delivery vector for the prevention of acute cell damage caused by ischemia. In particular, experiments were designed to examine the potential application of BacMam in delivering sod-2 using an in vitro model that closely mimicked renal I/R injury. We also evaluated whether ex vivo BacMam delivery of sod-2 in porcine kidneys might improve $\mathrm{I} / \mathrm{R}$ induced renal injury during cold preservation. 


\section{Material and Methods}

\section{Materials}

Antimycin A and 2-deoxy-D-glucose (2-deoxyglucose) were purchased from Sigma-Aldrich Co Ltd. Phosphate buffer saline (PBS) was supplied by GIBCO (Life Technologies Ltd). Foetal bovine serum (FBS) was supplied by Lonza Group Ltd and heat inactivated at $56^{\circ} \mathrm{C}$ for $30 \mathrm{~min}$.

\section{Cell Culture}

Insect cells were maintained at $28^{\circ} \mathrm{C}$ in Erlermeyer flasks (Corning) and glass Spinner flasks, respectively. Sf-9 cells were passaged before reaching their stationary phase using Sf9-S2 medium (PAA Laboratories Ltd); Sf-21 cells were maintained in TC100 (GIBCO, Life Technologies Ltd) supplemented with 10\% FBS. HK-2 cells were purchased from American Type Culture Collection (ATCC) and cultured in Keratinocyte Serum Free Medium (KSFM; GIBCO, Life Technologies Ltd.) supplemented with 2\% antibiotics (100 IU/mI penicillin, 100 $\mu \mathrm{g} / \mathrm{ml}$ streptomycin) at $37^{\circ} \mathrm{C} 5 \% \mathrm{CO}_{2}$.

\section{Plasmid construction and generation of BacMam vector}

The $\mathrm{pCl}$-neo plasmid, carrying the human cytomegalovirus immediate-early enhancer (CMVIE) promoter, was kindly provided by Prof Bermudez-Diaz (Oxford Brookes University, Oxford, UK). The pMA_SOD-2 plasmid was obtained from GeneArt $\circledast$ (Life Technologies Ltd); the sod-2 gene was synthesized based on the sequence of GenBank (accession number: Y00985). The baculovirus-based pBAC-1 plasmid was purchased from Novagen (EMD Biosciences, Inc).

To generate a pBAC-1 plasmid containing a CMV-IE promoter, the CMV-IE region was excised from pCl-neo with restriction endonucleases BgllI and Xhol. The CMV-IE promoter fragment was then ligated into the linearized pBAC-1 DNA, generating a pBAC-1_CMV transfer plasmid. Polymerase chain reaction (PCR) primers were designed for sod-2; (1) 
forward primer 5' AATTGAATTCGCACCATGTTGAGCCGGG 3' and (2) reverse primer 3' GTACCGAACGTTTTTCATTAGATCTTAAT 5'. The EcoRI (GAATTC) restriction site was added to the $5^{\prime}$ end of forward primer, while the Xbal (AGATCT) and terminator codon (TAA) were added to the $5^{\prime}$ end of the reverse primer. The sod-2 fragment was amplified from the pMA_SOD-2 plasmid by PCR using forward and reverse primers. The amplified sod-2 fragment and pBAC-1_CMV plasmid were digested simultaneously with EcoRI and Xbal and then ligated to create PBAC-1_SOD-2.

BacMam viruses were generated by homologous recombination between flashBAC ULTRA $^{\text {TM }}$ DNA (Oxford Expression Technologies Ltd) and pBAC-1_SOD-2 plasmid DNA, according to the manufacturer's instructions. The virus containing supernatant, AcCMV_SOD-2, was then harvested 5 days post-transfection and stored at $4^{\circ} \mathrm{C}$. FBS $(5 \%$ $v / v$ ) was added to the inoculum to improve stability during storage. High titre BacMam stock was obtained following further amplification of the viral stock in Sf-9 cells. AcCMV_SOD-2 virus was titrated by plaque assay ${ }^{40}$.

\section{Porcine kidney}

Experiments were performed in accordance with the principles of the Oxford Brookes University Ethics Committee. Porcine kidneys were obtained from a local abattoir. Organs were collected within 10 min after slaughter (22 week old large white pigs) and flushed in situ with soltran kidney perfusion fluid $(0.86 \% \mathrm{w} / \mathrm{v}$ potassium citrate, $0.82 \% \mathrm{w} / \mathrm{v}$ sodium citrate, $3.38 \% \mathrm{w} / \mathrm{v}$ mannitol and $1 \% \mathrm{w} / \mathrm{v}$ magnesium sulphate; Baxter). Kidneys were immediately transported in a biohazard bag, on ice (approximately $45 \mathrm{~min}$ ), to the laboratory for analysis.

\section{In vitro viral delivery}

HK-2 cells were seeded at a concentration of $0.3 \times 10^{6}$ cells $/ \mathrm{ml}$ and incubated overnight at $37^{\circ} \mathrm{C}$ in $5 \% \mathrm{CO}_{2}$. The following day, cells were transduced with BacMam viruses at an multiplicity of infection (MOI) of $150 \mathrm{pfu} / \mathrm{cell}$ and incubated at $37^{\circ} \mathrm{C}$ for $1 \mathrm{~h}$ in $5 \% \mathrm{CO}_{2}$. The 
virus inoculum was then replaced with growth culture medium and cells were returned to $37^{\circ} \mathrm{C}$ in $5 \% \mathrm{CO}_{2}$ for the required time.

\section{In vitro I/R injury model}

HK-2 cells were incubated in a phosphate saline buffer (PBS) containing $10 \mu \mathrm{M}$ antimycin $\mathrm{A}$ (a complex III inhibitor of mitochondrial electron transport) and $10 \mathrm{mM}$ 2-deoxyglucose (a nonmetabolizable isomer of L-glucose and a glycolysis inhibitor) for $60 \mathrm{~min}$ to induce in vitro ischemia injury ${ }^{41}$. Reperfusion was achieved in HK-2 cells by washing with PBS and then culturing in complete growth medium.

\section{Ex vivo viral delivery}

Flushed kidney organs were cannulated and perfused through the renal artery with $200 \mathrm{ml}$ of cold Belzer UW solution (Bridge to Life (Europe) Ltd), with or without BacMam. BacMam was concentrated using low speed-centrifugation and pellet dissolved in a physiological saline solution. During transduction, the $200 \mathrm{ml}$ Belzer UW solution was collected and recirculated using a low-speed peristaltic pump (10 rpm; Watson Marlow 505 S, Gemini BV). Kidneys were transduced for $24 \mathrm{~h}$. Tissue samples were collected using a $10 \mathrm{ml}$ syringe at 0,4 and $24 \mathrm{~h}$ post-transduction (hpt) and cells were immediately dissociated using collagenase (Sigma-Aldrich Co. Ltd).

\section{Measurement of cellular injury (ATP assay)}

Intracellular ATP levels were measured using a CellTitre-Glo® Luminescent Cell Viability Assay (Promega). Initially, the assay buffer and the lyophilized substrate were mixed together and left to equilibrate at room temperature for $20 \mathrm{~min}$. During the equilibration time, cells to be analysed were harvested by centrifugation and re-suspended in $100 \mu \mathrm{l}$ of $1 \mathrm{X}$ PBS. Subsequently, $100 \mu \mathrm{l}$ of the assay reagent were added into each tube and then mixed to release intracellular ATP into solution. After 5 min incubation, luminescence was read on 
a GloMax® 20/20 Luminometer (Promega). Data were normalized against the live cell number obtained using a trypan blue exclusion assay.

\section{Measurement of cytotoxicity (LDH assay)}

Cell viability was assessed by measuring the release of LDH into the culture media using CytoTox-ONE Homogeneous Membrane Integrity Assay (Promega). The CytoTox-ONE ${ }^{\mathrm{TM}}$ assay was carried out according to the manufactures guidelines. Briefly, mammalian cells to be evaluated were seeded into individual wells of a 96 well plate (Corning), treated with the appropriate chemical and incubated at $37^{\circ} \mathrm{C}$ for the required time. $2 \mu \mathrm{l}$ of lysis solution (supplied by the manufacturer) were added to the untreated cells for maximal LDH release. Untreated cells were used as negative control and a blank well served as a vehicle control. The cells were removed from $37^{\circ} \mathrm{C}$ and equilibrated at $22^{\circ} \mathrm{C}$ for $20 \mathrm{~min}$. CytoTox-ONE reagent was then added in an equal volume to the cell culture medium present in each well and shaken gently for $30 \mathrm{sec}$. Cells were incubated at $22^{\circ} \mathrm{C}$ for $10 \mathrm{~min}$ and the reaction was stopped by adding $50 \mu \mathrm{l}$ of stopping solution to each well. Fluorescence was recorded at an excitation wavelength of $560 \mathrm{~nm}$ and an emission wavelength of $590 \mathrm{~nm}$ using an Infinite $\circledast$ 200pro microplate reader (Tecan Group Ltd). Cytotoxicity values were obtained using the following calculation:

$$
\text { Cytotoxicity }(\%)=\frac{(\text { Experimental }- \text { Culture Medium Background })}{\text { (Maximum LDH Release }- \text { Culture Medium Background) }} \times 100
$$

\section{Western blot analysis}

Proteins separated on $12 \%$ SDS-PAGE gels were transferred onto a nitrocellulose membrane (GE Healthcare, UK) and analysed by western blot. Protein gels were incubated in transfer buffer (48 mM Tris, $39 \mathrm{mM}$ glycine, 20\% methanol and $1.3 \mathrm{mM} \mathrm{SDS}$ ) for 5 mins together with the nitrocellulose membrane and filter papers. Following the transfer, membranes were incubated in 5\% powdered milk dissolved in PBST (1X PBS containing 
$0.1 \%(\mathrm{v} / \mathrm{v})$ of Tween20) and agitated at room temperature for $1 \mathrm{~h}$ to prevent non-specific binding. Subsequently, membranes were treated with SOD-2 primary antibody for 60 min. Membranes were washed $3 \mathrm{X}$ with PBST and incubated with a secondary antibody conjugated with alkaline phosphatase for $60 \mathrm{~min}$. To remove any background, membranes were washed $3 \mathrm{X}$ with PBST. Bound proteins were visualized using alkaline phosphatase buffer $(100 \mathrm{mM}$ Tris- $\mathrm{HCl}(\mathrm{pH}=9.5), 100 \mathrm{mM} \mathrm{NaCl}$ and $5 \mathrm{mM} \mathrm{MgCl})$ containing $66 \mu \mathrm{l}$ of Nitro Blue tetrazolium (NBT) and $33 \mu \mathrm{l}$ of 5-Bromo-4-Chloro-3'-Indolyphosphate p-Toluidine (BCIP). The reaction was stopped by washing the membrane with in $\mathrm{dH}_{2} \mathrm{O}$ (distilled water) and any excess liquid was removed using filter paper. Band densitometry was carried out using GelQuantNET software (BiochemLabSolutions).

\section{Confocal microscopy}

Deparaffinised and hydrated kidney sections were permeabilised with $1 \mathrm{X}$ PBS containing $0.1 \%(\mathrm{v} / \mathrm{v})$ Triton $\mathrm{X}-100$. Tissue sections were blocked in $1 \mathrm{X}$ PBS-BSA prior to being incubated in the appropriate primary antibodies for $60 \mathrm{~min}$ at room temperature. Slides were washed three times in 1X PBS and incubated with the indicated secondary antibodies for 60 min at room temperature. Finally, any unbound antibody was removed using PBS and sections were mounted with Vectashield (Vector Laboratories) a mounting media containing the nuclear label 4',6-diamidino-2-phenylindole (DAPI). A clear nail-varnish was used to seal the mounted samples before being stored in the dark at $4^{\circ} \mathrm{C}$.

Confocal microscopy was performed on fixed tissue sections using a Zeiss LSM 510 Meta confocal system using X63 oil immersion lens. Multitrack analysis was carried out in order to analyse green fluorescence (488nm excitation, band pass filter 505-530nm), red and blue (DAPI) fluorescence $(543 \mathrm{~nm}$ excitation, long pass filter $560 \mathrm{~nm}$ for red, and 405 excitation, band pass filter 420-480nm for blue). Image data were analysed using LSM Image browser software. 


\section{Statistical analysis}

All data were analysed using GraphPad Prism version 5 for Windows (GraphPad Software Inc., USA) with results displayed as mean \pm SEM. Statistical analysis was performed using both one- and two-way ANOVA with a Bonferroni post-test that compared replicative mean values, unless otherwise stated. A p-value of $<0.05$ was considered statistically significant.

\section{Results}

\section{BacMam-mediated sod-2 expression in HK-2 cells}

Initial studies were performed to evaluate the relative BacMam transduction efficiency and the optimal transgene expression levels in human proximal tubule cells. HK-2 cells were transduced using different MOI with AcCMV_SOD-2 BacMam over a period of $96 \mathrm{~h}$ (hour). Results indicated that the viral dose played an important role in efficient transduction of HK-2 cells; MOI 150 resulted in minimal cytotoxicity and therefore was chosen as optimal for HK-2 cells (data not shown). Preliminary experiments showed that SOD-2 gradually increased from $0 \mathrm{~h}$ to $96 \mathrm{~h}$ following transduction with BacMam, as confirmed by western blot analysis (Figure 1). This was further confirmed by densitometry analysis, which indicated that SOD-2 expression increased from less than $1 \%$ to $11 \%$ within $24 \mathrm{~h}$ and subsequently to $23 \%, 32 \%$ and $34 \%$, at 48, 72 and 96 hpt (hour post-transduction), respectively (Figure 1). Because SOD-2 was optimally expressed between 72 and $96 \mathrm{~h}$ post-transduction, the $72 \mathrm{hpt}$ timepoint was chosen to assess the effect of BacMam-mediated gene delivery in an in vitro I/R injury model in HK-2 cells.

\section{Antimycin A and 2-Deoxyglucose/ATP-depletion injury in HK-2 cells}

To investigate the effects of I/R on renal cell injury, HK-2 cells were treated with antimycin A $(10 \mu \mathrm{M})$ and 2-deoxyglucose $(10 \mathrm{mM})$ to mimic the in vivo I/R response. As shown in Figure 2, exposure of HK-2 cells to chemicals caused an approximately two-fold decrease in intracellular ATP levels within 15 min of their addition compared to non-treated cells (filled 
squares vs. filled circles). This decrease in ATP levels was further induced in a timedependent manner; the cellular ATP content decreased by four-, nine- and six-fold following 30,45 and 60 min of chemical treatment, respectively $(p<0.001, N=3)$.

\section{Protective effect of sod-2 following ATP depletion-recovery in HK-2 cells}

To investigate whether BacMam-delivered sod-2 would protect against renal injury, HK-2 cells were transduced for $72 \mathrm{~h}$ with AcCMV_SOD-2 followed by exposure to antimycin $\mathrm{A}$ and 2-deoxyglucose ${ }^{41}$. As shown in Figure 3, HK-2 cells transduced with AcCMV_SOD-2, $72 \mathrm{~h}$ prior to I/R injury, exhibited a time-dependent increase in their intracellular ATP content (filled triangles) compared to cells transduced with an empty BacMam containing no gene, (AcCMV_NULL; filled square). BacMam-mediated sod-2 delivery showed an improved recovery of the cellular ATP content in the first $24 \mathrm{~h}$ of reperfusion (60\% increases) compared to AcCMV_NULL transduced/injured cells (40\%). ATP levels were significantly increased at 48 and $72 \mathrm{~h}$ after reperfusion in AcCMV_SOD-2 transduced/injured cells when compared to control cells and AcCMV_NULL transduced/injured cells $(p<0.001, \mathrm{n}=3)$.

Cytotoxicity was measured by the amount of LDH released from the cells into the media following the ATP depletion-recovery period. As shown in Figure 4, following $60 \mathrm{~min}$ of ischemia and $5 \mathrm{~min}$ of reperfusion, a significant increase in LDH release was detected $(p<0.05, \mathrm{n}=3)$ in AcCMV_NULL and AcCMV_SOD-2 transduced/injured cells (filled squares and triangles, respectively) compared to control cells (non-injured; filled circles). Prolonging the reperfusion phase, we observed that SOD-2 overexpression in HK-2 cells resulted in $80 \%, 75 \%$ and $70 \%$ reduction in $\mathrm{LDH}$ release following 24,48 and $72 \mathrm{~h}$ of reperfusion, respectively, compared to AcCMV_NULL transduced/injured cells (Figure 4). However, this time-dependent reduction in $\mathrm{LDH}$ release was only partial, since cytotoxicity in AcCMV_SOD-2 transduced injured cells, at $72 \mathrm{~h}$ after reperfusion, was still significantly higher compared to control cells (non-injured). 


\section{Effects of ex vivo BacMam-mediated sod-2 delivery against I/R-induced renal injury during cold preservation}

Initially, we demonstrated the feasibility of ex vivo BacMam gene transfer into a whole porcine kidney during cold preservation using a fluorescent marker. Immuno-detection showed Enhanced Green Fluorescent Protein (EGFP) specific staining in AcCMV_EGFP transduced kidney tissues but not in control kidneys (Figure 5).

Six fresh porcine kidneys (three left and three right) were obtained from a local abattoir, flushed in situ and then placed in a plastic bag surrounded by ice packs and immediately transported to the laboratory for analysis. Kidneys were divided into three experimental groups: group I (perfused control kidneys, no-virus), group II (AcCMV_EGFP transduced and perfused kidneys) and group III (AcCMV_SOD-2 transduced and perfused kidneys): each group contained a paired kidney (one left and one right) excised from the same pig. During the $24 \mathrm{~h}$ period of perfusion/transduction, triplicate tissue samples were collected from the left kidneys of each experimental group, weighed and enzymatically dissociated into single cells, using collagenase, for intracellular ATP analysis. As shown in Figure 6, ATP levels detected $0 \mathrm{~h}$ post-perfusion (hpp) in AcCMV_EGFP and AcCMV_SOD-2 transduced kidneys (0.4 and $0.3 \mathrm{nM}$, respectively) were not significantly different $(p>0.05, \mathrm{n}=3)$ compared to ATP concentrations detected in the non-transduced control group (0.3 nM). Significantly increased ATP levels were detected at 4 hpp in AcCMV_SOD-2 perfused/transduced kidney $(0.7 \mathrm{nM})$ compared to those detected in non-treated transduced kidney and AcCMV_EGFP transduced/perfused kidney, 0.25 and $0.24 \mathrm{nM}(p<0.001, \mathrm{n}=3)$, respectively; ATP levels in AcCMV_EGFP transduced kidney were not significantly different $(p>0.05, n=3)$ from the nontransduced control group (Figure 6, light grey bars vs. black bars).

Furthermore, a marked and significant increase $(p<0.001, \mathrm{n}=3)$ in intracellular ATP concentration was detected at $24 \mathrm{hpp}$ in AcCMV_SOD-2 transduced/perfused kidney tissue $(0.8 \mathrm{nM})$ compared to ATP levels observed in the experimental control group $0.5 \mathrm{nM}$ (Figure 
6, dark grey bars vs. black bars). In contrast, the intracellular ATP levels significantly decreased $(p<0.001, \mathrm{n}=3)$ in AcCMV_EGFP transduced/perfused kidney (0.2 nM) when compared to both AcCMV_SOD-2 and control experimental groups (Figure 6, light grey bars vs. dark grey bars).

\section{Discussion}

In order to study renal I/R injury, we initially performed in vitro experiments using proximal tubular cells. Western blot analysis confirmed BacMam transduction of HK-2 cells and demonstrated increasing SOD-2 expression over-time, as indicated by densitometry analysis (Figure 1). Although, a number of studies have described the delivery of sod-2 by viral vectors, mainly using adenovirus ${ }^{42,43}$, this is the first study demonstrating the feasibility of BacMam to deliver sod-2 to HK-2 cells.

The in vitro I/R injury model used in this study reproduced a number of key hallmark features of ischemia such as intracellular ATP depletion as well as reduction in tissue oxygen and glucose contents ${ }^{44}$. Previous studies demonstrated that the combination of antimycin $\mathrm{A}$ and 2-deoxyglucose produced $90 \%$ ATP depletion in HK-2 cells ${ }^{41,44,45}$. This is consistent with our findings where the combination of antimycin A and 2-deoxyglucose induced cellular injury; indeed, the intracellular ATP levels were significantly reduced within 30 min of treatment (Figure 2). Likewise, previous studies have demonstrated that the ATP/glucose depletion model of anoxia/ ischemia Belzer UW dramatically depleted intracellular ATP levels to 1-4\% of control cells ${ }^{41,46}$, as observed in our experiments. Kevin et al. ${ }^{47}$ demonstrated that treatment of Madin-Darby canine kidney (MDCK) cells with $10 \mu \mathrm{M}$ antimycin A dissolved in a Dulbecco's phosphate-buffered saline (DPBS glucose-free), caused a rapid decrease in the intracellular level of ATP; by $2 \mathrm{~h}$ levels were below the limit of detection and essentially zero after $5 \mathrm{~h}$.

Having established and validated a cell culture model of renal injury, we next determined whether I/R injury could be either reduced or prevented in HK-2 cells by the delivery of sod-2 
via BacMam. SOD-2 transduced-injured cells showed a trend of increasing intracellular ATP levels over-time $(20 \%$ to $140 \%)$, with ATP recovery observed at $72 \mathrm{~h}$, compared to AcCMV_NULL transduced/injured cells (19\% to $86 \%$; Figure 3$)$. Since the intracellular ATP content is essential for maintaining normal cellular functions as well as cellular integrity, it should be noted that any increase, even small, in ATP content during I/R injury might contribute to long-term cellular recovery. Although ATP recovery was observed at $72 \mathrm{~h}$ postreperfusion in AcCMV_SOD-2 transduced/injured cells, the increase in ATP levels, observed between 6 and $48 \mathrm{~h}$ post-reperfusion, also exerted a protective effect against I/R injury. These observations were in agreement with published models of renal I/R injury in cells overexpressing SOD $-2^{35}$. Cruthirds et al. ${ }^{48}$ demonstrated that after stable transfection, normal renal kidney (NRK) cells producing recombinant SOD-2 were protected against I/R injury induced by ATP depletion. Koo et al. ${ }^{43}$ generated a stable alveolar epithelial cell line overexpressing SOD-2; they demonstrated that the overexpression of SOD-2 significantly protected cells within the first $48 \mathrm{~h}$ of hyperoxia exposure and significantly increased the number of viable cells.

Exposure of transduced HK-2 cells to I/R injury ( $0 \mathrm{hpr}$ ) resulted in an increased release of LDH compared to control cells (Figure 4). However, time-course analysis of I/R-induced LDH release in AcCMV_SOD-2 injured HK-2 cells showed a decrease in LDH levels at 24, 48 and 72 hpr compared to AcCMV_NULL transduced/injured cells, which remained higher during the $72 \mathrm{~h}$ of reperfusion (Figure 4). These findings were consistent with the ATP data (Figure 3).

A number of reports have demonstrated that the primary cause of ischemia in the kidneys is due to clamping of the renal arteries, which may occur during the surgical procedure of renal transplantation ${ }^{49}$. In particular, strategies for organ preservation are fundamentally based on the prevention or suppression of the metabolic changes that occur during I/R injury ${ }^{49}$. 
We observed EGFP expression following ex vivo transduction into porcine kidneys (Figure 5, panel $\mathrm{A} 2)^{50}$ and to our knowledge, this is the first evidence of ex vivo sod-2 delivery in kidneys using BacMam. We also tested whether augmentation of sod-2 might improve I/R induced renal injury during cold preservation. A progressive and significant increase in ATP concentration was observed, between 4 and $24 \mathrm{hpp}$, in kidneys overexpressing sod-2 but not in control kidneys (Figure 6, dark grey bars vs. black bars). Results also showed a significant negative effect in AcCMV_EGFP transduced kidney (Figure 6, light grey bars), with markedly reduced levels of intracellular ATP at $24 \mathrm{hpp}$ compared to untreated AcCMV_SOD-2 transduced kidneys (Figure 6); these data were in line with results of the in vitro experiments (unpublished data).

These observations are consistent with those made by Land et al. ${ }^{51}$ that sod exerts a beneficial effect against renal I/R injury through its antioxidant action. Furthermore, our study suggested a potential protective effect of ex vivo BacMam-mediated delivery of sod-2 against long cold kidney preservation injury. However, there are clearly limitations inherent to the ex vivo cold perfusion model used in this experiment that need to be addressed in future studies. The main limitation is represented by the short lifespan of the organ perfused and knowing when valid data can be extracted during this time ${ }^{52}$. Ex vivo cold perfusion models are also restricted by the fact that hypothermic preservation can significantly decrease the organ's metabolic functions, which can preclude full recovery ${ }^{53}$.

In conclusion, the present findings confirm the feasibility of using BacMam as a gene delivery vector to prevent renal deterioration after tissue $\mathrm{I} / \mathrm{R}$ injury in vitro as well as in ex vivo isolated perfused porcine kidney. In particular, this study showed the beneficial effect of BacMam-delivered sod-2, which protected kidney from further renal injury after ischemia.

\section{Acknowledgements}

The authors are grateful to Dr Susan Brooks for helpful discussions on histopathology. We also thank $\mathrm{Dr}$ John Runions and $\mathrm{Dr}$ Sarah Irons for their assistance with confocal 
microscopy. This study was funded by Oxford Brookes University studentship and partially supported by Oxford Expression Technologies Ltd, Oxford, UK.

\section{Author Disclosure Statement}

The authors declare no conflicting interests.

\section{References}

1. Salvadori M, Rosso G, Bertoni E. Update on ischemia-reperfusion injury in kidney transplantation: Pathogenesis and treatment. World J Transplant. 2015 Jun 24;5(2):5267.

2. Versteilen, A. M., Di Maggio, F., Leemreis, J. R., et al. Molecular mechanisms of acute renal failure following ischemia/reperfusion. Int J Artif Organs, 2004; 27(12), 1019-29.

3. Cassis, P., Solini, S., Azzollini, N., et al. An unanticipated role for survivin in organ transplant damage. Am J Transplant, 2014; 14(5), 1046-60.

4. Lee HT, Gallos G, Nasr SH, et al. A1 adenosine receptor activation inhibits inflammation, necrosis, and apoptosis after renal ischemia-reperfusion injury in mice. J Am Soc Nephrol 2004; 15:102-111

5. Yang, B., Jain, S., Pawluczyk, I. Z., et al. Inflammation and caspase activation in longterm renal ischemia/reperfusion injury and immunosuppression in rats. Kidney Int, 2005; 68(5), 2050-67.

6. Mister M., Noris M., Szymczuk J., et al. Propionyl-I-carnitine prevents renal function deterioration due to ischemia/reperfusion. Kidney International 2002; 61, 1064-1078.

7. Vajdová K., Graf R., and Clavien PA. ATP-Supplies in the Cold-Preserved liver: A Long-Neglected Factor of Organ Viability. Hepatology. Dec; 2002; 36(6):1543-52.

8. Atchison N., Swindlehurst G., Papas KK., et al. Maintenance of ischemic $\beta$ cell viability through delivery of lipids and ATP by targeted liposomes. Biomater Sci. 2014; Apr $1 ; 2(4): 548-559$ 
9. Liu S., Soong Y., Seshan S. V., et al. Novel cardiolipin therapeutic protects endothelial mitochondria during renal ischemia and mitigates microvascular rarefaction, inflammation, and fibrosis. American Journal of Physiology - Renal Physiology 2014; Vol. 306 no. 9.

10. Hotter G, Palacios L, Sola A. Low O2 and high CO2 in LLC-PK1 cells culture mimics renal ischemia-induced apoptosis. Lab Invest. 2004 Feb;84(2):213-20.

11. Xie J, Guo Q. Apoptosis antagonizing transcription factor protects renal tubule cells against oxidative damage and apoptosis induced by ischemia-reperfusion. J Am Soc Nephrol. 2006 Dec;17(12):3336-46.

12. Mueller S, Millonig G, Waite GN. The GOX/CAT system: a novel enzymatic method to independently control hydrogen peroxide and hypoxia in cell culture Adv Med Sci. 2009;54(2):121-35. doi: 10.2478/v10039-009-0042-3.

13. . Beauchamp P, Richard V, Tamion F, Lallemand F, Lebreton JP, Vudry H, Daveau M, Thuillez C. Protective effects of preconditioning in cultured rat endothelial cells. Effects on neutrophil adhesion and expression of ICAM-1 after anoxia and reoxygenation. Circulation, 1999. 100:541-546

14. Kurianand GA, Pemaih B. Standardization of in vitro Cell-based Model for Renal Ischemia and Reperfusion Injury. Indian J Pharm Sci. 2014 Jul-Aug; 76(4): 348-353.

15. Quinlan, C. L., Perevoshchikova, I. V., Hey-Mogensen, M., etal. Sites of reactive oxygen species generation by mitochondria oxidizing different substrates. Redox Biol, 2013; 1(1), 304-12.

16. Zelko, I.N., Mariani, T.J. and Folz, R.J. Superoxide Dismutase multigene family: a comparison of the Cu-Zn-SOD (SOD1), Mn.SOD (SOD2) and EC-SOD (SOD3) gene structures, evolution and expression. Free Radic Biol Med 202; 33: 337-349.

17. Barra, D., Schinina, M. E., Simmaco, M., et al. The primary structure of human liver manganese superoxide dismutase. J Biol Chem, 1984; 259(20), 
18. MacMillan-Crow LA, Crow JP, Kerby JD, et al. Nitration and inactivation of manganese superoxide dismutase in chronic rejection of human renal allografts. Proceedings of the National Academy of Sciences of the USA 1996;

19. Macmillan-Crow L.A. and Cruthirds, D.L. Manganese Superoxide Dismutase in Disease. Free Radical Research, 2001; 34, 325-336.

20. Aguirre, J D; Culotta, V C. Battles with iron: manganese in oxidative stress protection. J Biol Chem 2012; 287(17):13541-8

21. Cruthirds DL, Novak L, Akhi KM. Mitochondrial targets of oxidative stressduring renal ischemia/reperfusion. Arch. Biochem. Biophys., 2003; 412: 27-33

22. Kasahara, E., Lin, L. R., Ho, Y. S. SOD2 protects against oxidation-induced apoptosis in mouse retinal pigment epithelium: implications for age-related macular degeneration. Invest Ophthalmol Vis Sci, 2005; 46(9), 3426-34.

23. Fukui, M. and Zhu, B. T. Mitochondrial superoxide dismutase SOD2, but not cytosolic SOD1, plays a critical role in protection against glutamate-induced oxidative stress and cell death in HT22 neuronal cells. Free Radic Biol Med, 2010; 48(6), 821-30.

24. Walther, W., Stein, U. Viral vectors for gene transfer: a review of their use in the treatment of human diseases. Drugs. 2000;60(2):249-71.

25. Collins M, Thrasher A. Gene therapy: progress and predictions. Proc Biol Sci. 2015 Dec 22;282(1821):20143003. doi: 10.1098/rspb.2014.3003.

26. Fornwald J. A., Lu Q., Wang D. Gene expression in mammalian cells using BacMam, a modified baculovirus system. Methods Mol Biol. 2007; 388:95-114.

27. Wickham, T. J., R. R. Granados, H. A. Wood, D. A. Hammer, and M. L. Shuler. General analysis of receptor-mediated viral attachment to cell surfaces. Biophys. J. 1990, 58:1501-1516.

28. Blissard, G. W., and J. R. Wenz. Baculovirus gp64 envelope glycoprotein is sufficient to mediate pH-dependent membrane fusion. J. Virol. 1992. 66:6829-6835. 
29. van Loo ND, Fortunati E, Ehlert E, Rabelink M, Grosveld F, Scholte BJ. Baculovirus infection of nondividing mammalian cells: mechanisms of entry and nuclear transport of capsids. J Virol. 2001 Jan;75(2):961-70.

30. Wheeler, M. D., Katuna, M., Smutney, O. M. et al. Comparison of the effect of adenoviral delivery of three superoxide dismutase genes against hepatic ischemia-reperfusion injury. Hum Gene Ther, 2001; 12(18), 2167-77.

31. Ayres, M. D., Howard, S. C., Kuzio, J. The complete DNA sequence of Autographa californica nuclear polyhedrosis virus. Virology, 1994; 202(2), 586-605.

32. Condreay, J. P., Witherspoon, S. M., Clay, W. C. Transient and stable gene expression in mammalian cells transduced with a recombinant baculovirus vector. Proc Natl Acad Sci U S A, 1999; 96(1), 127-32.

33. Paul A, Hasan A, Rodes L, Sangaralingam M, Prakash S. Bioengineered baculoviruses as new class of therapeutics using micro and nanotechnologies: principles, prospects and challenges. Adv Drug Deliv Rev. 2014 May;71:115-30.

34. Abe T, Hemmi H, Miyamoto H, Moriishi K, Tamura S, Takaku H, Akira S, Matsuura Y. Involvement of the Toll-like receptor 9 signaling pathway in the induction of innate immunity by baculovirus. J. Virol. 2005, 79:2847-2858.

35. Boulaire J, Zhao Y, Wang S. Gene expression profiling to define host response to baculoviral transduction in the brain. J. Neurochem. 2009, 109:1203-1214.

36. Han Y, Niu M, An L, Li W. Upregulation of proinflammatory cytokines and NO production in BV-activated avian macrophage-like cell line (HD11) requires MAPK and NF-kappaB pathways. Int. Immunopharmacol. 2009, 9:817-823.

37. Abe T, Kaname Y, Wen X, Tani H, Moriishi K, Uematsu S, Takeuchi O, Attenuation of Transgene Expression by Baculovirus February 2014 Volume 88 Number 4

38. Ono C, Ninomiya A, Yamamoto S, Abe T, Wen X, Fukuhara T, Sasai M, Yamamoto M, Saitoh T, Satoh T, Kawai T, Ishii KJ, Akira S, Okamoto T, Matsuura Y. Innate immune response induced by baculovirus attenuates transgene expression in mammalian cells. $\mathrm{J}$ Virol. 2014 Feb;88(4):2157-67. 
39. Hitchman RB, Murguía-Meca F, Locanto E, Danquah J, King LA. Baculovirus as vectors for human cells and applications in organ transplantation. J Invertebr Pathol. 2011 Jul;107 Suppl:S49-58.

40. King, L. A., Possee, R. D. The Baculovirus Expression System: A laboratory Guide. Chapman and Hall, London, 1992; pp.17-73.

41. Lee H.T., Emala C.W. Preconditioning and adenosine protect human proximal tubule cells in an in vitro model of ischemic injury. J Am Soc Nephrol 2002; 13: 2753-2761.

42. Pérez M.J., Cederbaum A.I. Adenovirus-mediated expression of $\mathrm{Cu} / \mathrm{Zn}$ - or $\mathrm{Mn}$ superoxide dismutase protects against CYP2E1-dependent toxicity. Hepatology, 2003; 38(5):1146-58.

43. Koo H.C., Davis J.M., Li Y., Hatzis D. Effects of transgene expression of superoxide dismutase and glutathione peroxidase on pulmonary epithelial cell growth in hyperoxia. Am J Physiol Lung Cell Mol Physiol 2005; 288(4):L718-26.

44. Xie J., Guo Q. Par-4 is Novel Mediator of Renal Tubule Cell Death in Models of Ischemia-Reperfusion Injury. Am. J. Physiol. Renal Physiol 2007.

45. Iwata S., Hori T., Sato N., et al. Thiol-mediated redox regulation of lymphocyte proliferation: possible involvement of adult T cell leukemia-derived factor and glutathione in transferrin receptor expression. J Immunol 1994; 152: 5633-5642

46. Breggia A.C., Himmelfarb J. Primary mouse renal tubular epithelial cells have variable injury tolerance to ischemic and chemical mediators of oxidative stress. Oxid Med Cell Longev. 2008;1(1):33-8.

47. Kevin T. B., Tatsuo T., Sanjay K. N.Selective degradation of E-cadherin and dissolution of E-cadherin-catenin complexes in epithelial ischemia. Am J Physiol Renal Physiol 1999; 278: F847-F852, 2000

48. Cruthirds D.L., Saba H., MacMillan-Crow L.A. Overexpression of manganese superoxide dismutase protects against ATP depletion-mediated cell death of proximal tubule cells. Arch Biochem Biophys 2005;437(1):96-105. 
49. Watson C. J. E., Dark J. H. Organ transplantation: historical perspective and current practice. Br. J. Anaesth. 2012; 108 (suppl 1): i29-i42.

50. Murguía-Meca F., Plata-Muñoz J.J., Hitchman R.B., et al. Baculovirus as delivery system for gene transfer during hypothermic organ preservation. Transpl Int. 2011;24(8):820-8.

51. Land W., Schneeberger H., Schleibner S., et al. The beneficial effect of human recombinant superoxide dismutase on acute and chronic rejection events in recipients of cadaveric renal transplants. Transplantation 1994;57(2):211-7.

52. Ong S.L., Gravante G., Metcalfe M.S. History, ethics, advantages and limitations of experimental models for hepatic ablation. World J Gastroenterol 2013; 14;19(2):147-54.

53. St Peter S. D., Imber C. J., Friend P. J. Liver and kidney preservation by perfusion. Lancet 2002; 359: 604-13 


\section{Figure Legends}

Figure 1. MnSOD expression in HK-2 cells. BacMam virus containing MnSOD gene under control of the cytomegalovirus immediate early (CMV-IE) promoter, was used to transduce HK-2 cells at a multiplicity of infection (MOI) of 150. Transduced HK-2 cells were harvested at $0,24,48,72$ and 96 and cell lysates were western blotted onto nitrocellulose membrane using anti-MnSOD antibody. Lanes 0 to 96 represent time posttransduction. The arrow refers to MnSOD proteins at approximately 23 kiloDalton (kDa).

Figure 2. Time-course of ATP decay during simulated ischaemia in HK-2 cells. Control cells were incubated in KSF medium. HK-2 cells were exposed to antimycin A $(10 \mu M)$ and 2-Deoxy-D-Glucose $(10 \mathrm{mM})$. ATP levels were determined every $15 \mathrm{~min}$ using a Cell TitreGlo assay (Promega). Error bars represent mean $\pm \operatorname{SEM}(n=3) p<0.001$ indicated a significant difference between control and injured cells (two-way ANOVA).

Figure 3. Intracellular ATP levels in I/R injured HK-2 cells. HK-2 cells were transduced with either AcCMV_NULL (filled squares) or AcCMV_MnSOD (filled triangles) at MOI 150. At $72 \mathrm{hpt}$ transduced HK-2 cells were subjected to 60 min of simulated ischemia. Intracellular ATP levels were quantified over-time. Error bars represent mean \pm SEM $(n=3) ;{ }^{*} p<0.001$ indicated a significant difference between control cells (non-injured) and AcCMV_MnSOD transduced injured cells.

Figure 4. Effect of ACCMV_MnSOD on the release of lactate LDH from injured HK-2 cells. Cells were transduced with either AcCMV_NULL (filled squares) or AcCMV_MnSOD (filled triangles) at MOI 150 for $72 \mathrm{~h}$ before being subjected to $60 \mathrm{~min}$ of simulated ischemia following reperfusion. AcCMV_MnSOD causes a time-dependent reduction of $\mathrm{LDH}$ release caused by antimycin A and 2-deoxyglucose. Data are 
expressed as mean \pm SEM $(n=3) ;{ }^{*} p<0.001$ indicated a significant difference between control cells (filled circles) and AcCMV_MnSOD transduced injured cells (filled triangles).

Figure 5. Egfp expression in sections of control and BacMam transduced kidneys. Kidneys were incubated in BELZER UW® solution supplemented with or without AcCMV_EGFP virus and incubated at $4^{\circ} \mathrm{C}$ for $24 \mathrm{~h}$. (A1) Control and (A2) BacMam transduced kidneys. The kidney sections were fixed with paraformaldehyde, immunofluorescent stained for egfp primary antibody and then labelled red with Alexa Fluor®-568 conjugated phalloidin secondary antibody. Tissues were analysed by confocal microscopy using an oil immersion x63 objective. Scale bar=10 $\mu \mathrm{m}$.

\section{Figure 6. Intracellular ATP levels in non-transduced and BacMam} perfused/transduced porcine kidneys. Tissue kidney samples were collected overtime from control groups (no virus; black bars), AcCMV_EGFP (light grey bars) and AcCMV_MnSOD (dark grey bars) transduced kidney groups. Kidney samples were enzymatically dissociated in single cells and ATP level measured over-time. Error bars represent mean \pm SEM $(n=3) ;{ }^{*} p<0.001$ indicated a significant difference between the ATP levels of perfused control experimental group and AcCMV_MnSOD perfused/transduced kidneys. 


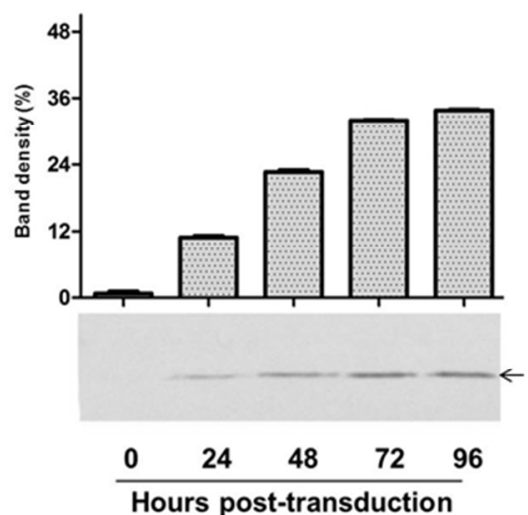

$216 \times 121 \mathrm{~mm}(96 \times 96$ DPI $)$ 


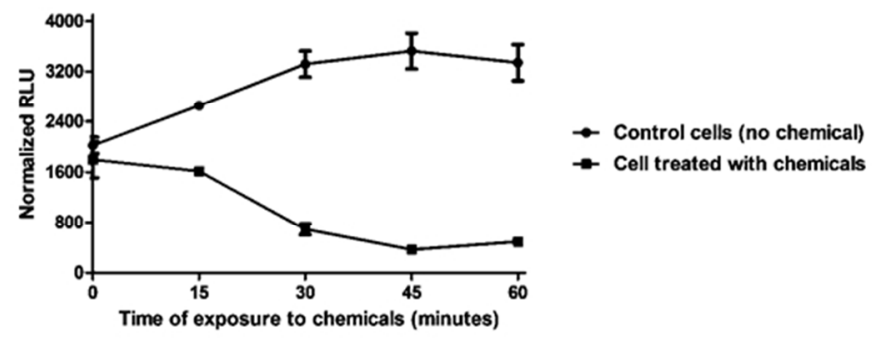

$216 \times 121 \mathrm{~mm}(96 \times 96 \mathrm{DPI})$ 
$\rightarrow$ Control cells (non-injured)

\section{- AcCMV_NULL transduced injured cells}

- ACCMV_MnSOD transduced injured cells

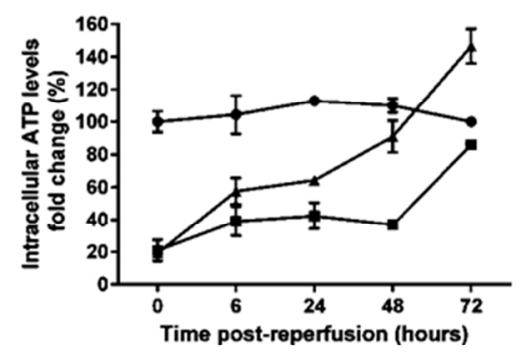

$216 \times 121 \mathrm{~mm}(96 \times 96 \mathrm{DPI})$ 


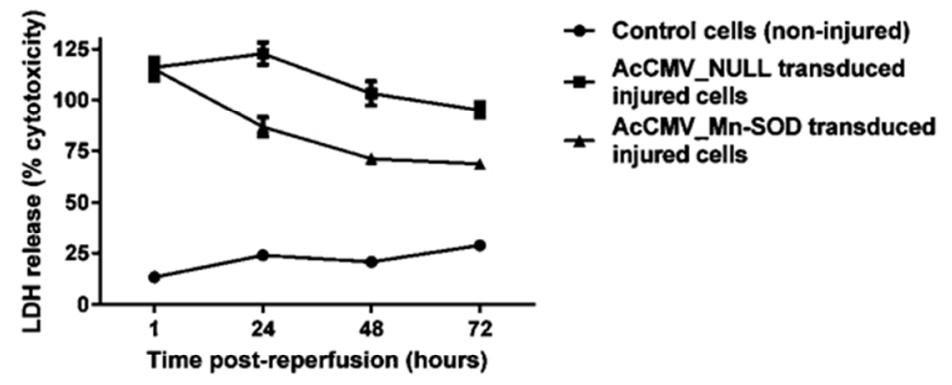

$216 \times 121 \mathrm{~mm}(96 \times 96 \mathrm{DPI})$ 


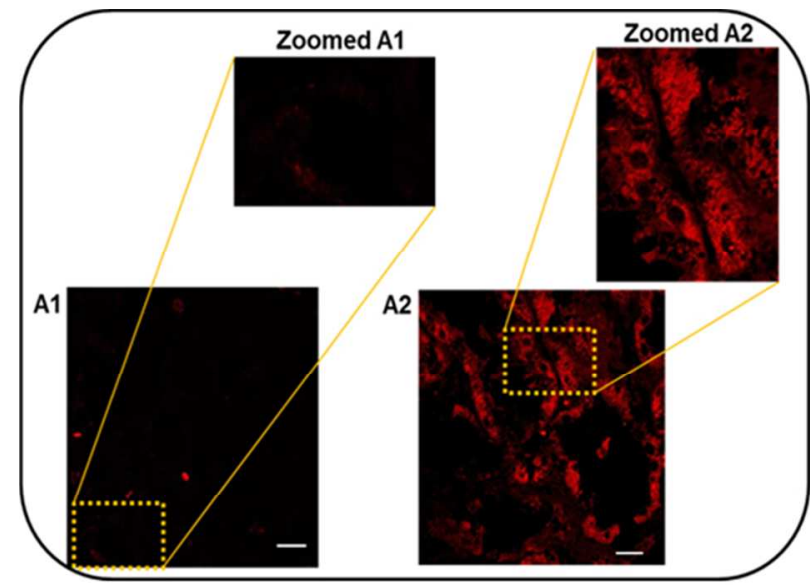

$216 \times 121 \mathrm{~mm}(96 \times 96 \mathrm{DPI})$ 


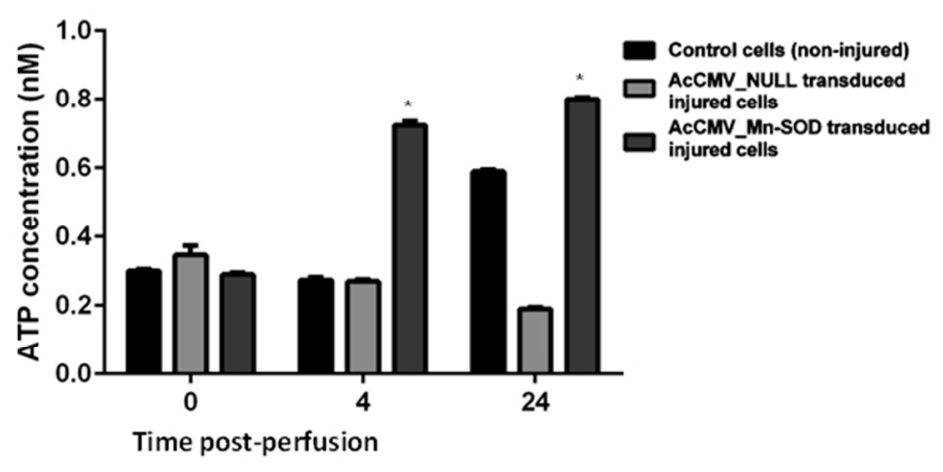

$216 \times 121 \mathrm{~mm}(96 \times 96 \mathrm{DPI})$ 